\title{
A Window on the Ultra-High Energy Universe
}

\author{
Pasquale Blasi \\ INAF/Osservatorio Astrofisico di Arcetri \\ Largo E. Fermi, 5 - I50125 Firenze, Italy
}

\begin{abstract}
Cosmic rays with energy around and in excess of $10^{20} \mathrm{eV}$ have been detected. Despite the uncertainties associated with the difficult measurements of extremely low fluxes (about a particle per square kilometer per century) the same existence of such high energy cosmic rays raises important questions that deserve an appropriate answer. I briefly summarize here what are these questions and how to look for the answers.
\end{abstract}

\section{The highest energy particles}

In the eighty years of history of cosmic rays, there has been a constant search for the end of the cosmic ray spectrum. It has long been thought that this end of the spectrum would be determined by the highest energy that cosmic accelerators might be able to achieve. Despite this continuous search, no end was found. In 1966, right after the discovery of the cosmic microwave background (CMB), it was understood (Greisen 1966, Zatsepin and Kuzmin 1966) that high energy protons would inelastically scatter the photons of the CMB and produce pions. The concept of GZK cutoff was introduced and for the first time the end of the cosmic ray spectrum was associated with a physical process rather than with speculations on the nature of the accelerators. For the first time, the end of the cosmic ray spectrum was predicted to be at a well defined energy, around $10^{20} \mathrm{eV}$, where the so-called photopion production starts to be kinematically allowed. Forty years later, we are still seeking a confirmation that the cosmic ray spectrum has in fact such a flux suppression, although we understood that it is not a sharp cutoff. The two largest experiments currently operating in the energy range of interest, namely AGASA and HiRes, appear to have discrepant results in the highest energy end of the spectrum. While the data collected by the former appear to be consistent with the extension of the lower energy spectrum, the latter experiment suggests that the GZK feature is present in the data. In fact, it has been shown by De Marco, Blasi \& Olinto (2003) that this discrepancy is only at the level of $\sim 2.6 \sigma$ and even less significant (about $2 \sigma$ ) if a systematic error in the energy determination is introduced, something which in fact would also explain an offset between the fluxes as measured by the two experiments. The required systematic error is of $30 \%$, which can be shared between the two experiments, being compatible with the published estimates of such errors. More and better data are required to say the final word about the detection of the GZK suppression. 


\section{A short summary of the observations}

The GZK feature should not be considered as the whole of the problem of UHECRs. Many questions remain open, whether the GZK feature is discovered or not. In this section I briefly summarize the main pieces of the puzzle of UHECRs:

Isotropy: The directions of arrival of the events at energies above $\sim 4 \times 10^{19}$ $\mathrm{eV}$ appear isotropically distributed in the sky. No immediate association with local structures (galactic disc, supergalactic plane) arises from the data.

Missing identification: No association of the observed events with known powerful nearby sources has been found. It is important to realize that this may be a problem only for the highest energy events, with energy higher than $10^{20}$ $\mathrm{eV}$, for which the loss length is small and the sources are forced to be closeby. Even at $4 \times 10^{19} \mathrm{eV}$ the loss length is comparable with the size of the universe and it is therefore difficult to find a counterpart, in particular because of the poor angular resolution of current experiments.

Small Scale Anisotropies: The AGASA data show several doublets and triplets of events on angular scales comparable with the resolution of the instrument. The statistical significance of these multiplets is still the subject of some debate [see for instance (Finley and Westerhoff, 2003)], but if confirmed as not just the result of statistical fluctuations they could in fact represent the first evidence that UHECRs are accelerated in astrophysical point sources. This evidence would point against most so-called top-down models, in which the emission is truly diffuse.

The composition: At the highest energies the information about the chemical composition is so far very poor. A reanalysis of the Haverah Park inclined showers allowed to constrain the fraction of gamma rays at energy larger than $4 \times 10^{19} \mathrm{eV}$ to about $50 \%$ (Ave, et al. 2002). This is unfortunately still too weak a limit to disprove most top-down models.

\section{What are the sources of UHECRs?}

This is a clear example of a question that will long survive the detection or the lack of detection of the GZK feature. Many possibilities have been put forward in the literature but at present there is no clear indication in favor of one specific model. Rather than briefly listing a bunch of models that may in principle be responsible for the acceleration of UHECRs, I prefer here to discuss some lines of thought that might in fact bring us toward the identification of one or more classes of sources. Two problems have made this step very difficult: first, the lack of any identification of counterparts for the highest Fly's Eye event at energy $3 \times 10^{20} \mathrm{eV}$ (Bird, et al. 1993); second, the fact that from the energy budget in the form of UHECRs per unit volume it is not easy to know whether many sources with low luminosity or a few powerful sources are responsible for the acceleration of UHECRs. I will call this the degeneracy problem.

For the first obstacle, the situation has not changed in the last decades: on the other hand one should think of this situation as similar to what happened for gamma ray bursts (GRBs). Until a few years ago, GRBs were defined as gamma ray flashes with no counterpart at any wavelengths. After the identification 
of the afterglows, the situation has completely changed and much has been understood on the nature of the sources, although a complete picture is still lacking.

The degeneracy problem on the contrary has changed appreciably during the last few years, after the discovery of the small scale anisotropies in the directions of arrival of UHECRs. Although the statistical significance of these anisotropies is still matter of debate, their appearance forced us to think of them as a powerful tool to probe a distribution of point sources of UHECRs. Several authors pointed out that the number density of sources is related to the number of doublets and triplets of events, or in other words, to the two-point correlation function. Blasi \& De Marco (2003) showed that current observations favor sources with space density around $10^{-6}-10^{-5} \mathrm{Mpc}^{-3}$, corresponding to an integrated luminosity above $10^{19} \mathrm{eV}$ of $10^{42}-10^{43} \mathrm{erg} \mathrm{s}^{-1}$. This is the first time that the degeneracy between source density and luminosity of the single sources gets broken. It does not imply that the sources of UHECRs have been identified, but certainly selects some classes of sources. In (Blasi \& De Marco, 2003) it was shown that future experiments such as Auger and EUSO have the potential to accumulate enough statistics to improve on this front significantly. In fact, for the range of source densities mentioned above, it should be possible even to measure the spectrum of single nearby sources of UHECRs and infer their distance (Blasi \& De Marco 2003).

The unambiguous detection of small scale anisotropies in the distribution of arrival directions would represent a strong argument in favor of astrophysical point sources and therefore against the alternative top-down scenarios.

\section{What is the chemical composition of UHECRs?}

The measurement of the chemical composition of UHECRs is difficult because of the low statistics of events and because of the large fluctuations in the shower development. Upcoming experiments such as Auger and EUSO represent clear improvements on the present situation, although the measurement of the composition will remain challenging.

The chemical composition represents a discriminant factor among different models for the generation of UHECRs. In some acceleration scenarios (e.g. Blasi, Epstein, \& Olinto 2000) the composition is expected to be dominated by iron nuclei. On the other hand, in the context of top-down models, an appreciable fraction of the cosmic rays is expected to be in the form of gamma rays. More specifically, the spectrum generated in the case of decay of supermassive relic particles clustered in the galactic halo is expected to contain predominantly gamma rays. More dependent upon the details of the propagation is the composition in the case of topological defects, where absorption of gamma rays upon the diffuse extragalactic radio background becomes important. This background is unfortunately poorly known, because of the impossibility to measure this background from within the Galaxy, due to free-free absorption. The detection of gamma rays at the highest energies would be a smoking gun in favor of top-down scenarios and an unprecedented discovery of new physics in the early universe. 


\section{References}

Ave, M., Hinton, J.A., Vazquez, R.A., Watson, A.A., Zas, E. 2002, Phys. Rev., D65, 063007

Bird, D.J., et al 1993, Phys. Rev. Lett., 71, 3401

Blasi, P. \& De Marco, Astropart. Phys., in press (preprint astro-ph/0307067)

Blasi, P., Epstein, R.I., \& Olinto, A.V. 2000, ApJ Lett., 533, 123

De Marco, D., Blasi, P., \& Olinto, A.V. 2003, Astropart. Phys., 20, 53

Finley, C.B., \& S. Westerhoff 2003, preprint astro-ph/0309159

Greisen, K. 1966, Phys. Rev. Lett., 16, 748

Zatsepin, G.T., \& and Kuzmin, V.A. 1966, Sov. Phys. JETP Lett., 4, 78 